\title{
Kooperationen sind Tauschgeschäfte
}

\section{Wie man Unternehmen als Partner gewinnt}

\author{
Helene Reuther
}

Helene Reuther ist Geschäftsführerin von »morgenwelt «, eine Agentur für Sozialmarketing in Köln.

E-Mail H.Reuther@morgenwelt.net
Soziale Organisationen, die Unternehmen als Kooperationspartner gewinnen wollen, müssen sich zunächst über den Nutzen klarwerden, den sie diesen bieten können. Die erfolgreiche »Tauschwährung " für Unternehmen heißt dann Kommunikation.

Sie planen ein neues Projekt und brauchen finanzielle Unterstützer, um es realisieren zu können. Am besten geeignet erscheint Ihnen dafür ein Kooperationspartner aus der Wirtschaft. Aber wie findet man das passende Unternehmen? Wie geht man die Suche an? Getreu dem Motto "Der Wurm muss dem Fisch schmecken und nicht dem Angler " gilt es primär, die Motive eines Sponsors zu verstehen. Sich vorstellen zu können, wie die Menschen in einem Unternehmen denken und welche Fragen sie bewegen, ist ein Schlüssel zum Erfolg.

\section{Die Motive und Ziele von Unternehmen}

Kooperationen sind Tauschgeschäfte. Unternehmen geben nicht uneigennützig, sie versprechen sich einen Nutzen, wenn sie eine soziale Organisation mit Geld, Sachleistungen oder Know-how unterstützen. In der Regel verfolgen sie mit ihrem Engagement strategische Marketingund Kommunikationsziele. Die »Tauschwährung « für Unternehmen heißt Kommunikation. Das kann sowohl die interne als auch die externe Kommunikation sein. Attraktiv aus Unternehmenssicht ist daher ein Projekt, welches

- das Image verbessert (sowohl intern wie extern)

- das Ansehen am Standort positiv beeinflusst

- die Identifikation und Motivation von Mitarbeiterinnen und Mitarbeitern stärkt

- neue Zielgruppen anspricht

- den Kontakt zu Kunden und Meinungsbildnern intensiviert
- den Bekanntheitsgrad steigert oder stabilisiert

- den Umsatz und den Verkauf von Produkten steigert

Je klarer die eigenen Möglichkeiten und Leistungen (Stärken und Schwächen) analysiert und beschrieben sind, desto deutlicher können die Erwartungen eines potenziellen Sponsors erfüllt werden und desto erfolgreicher wird die Kooperation sein. Bevor eine Organisation also konkret auf die Suche nach einem Partner aus der Wirtschaft geht, muss geklärt sein, welchen Nutzen die Organisation oder das Projekt bieten können und welches Unternehmen dazu passt.

\section{Die gezielte Suche nach Firmenpartnern}

Wenn Sie nicht in der glücklichen Lage sind, bereits Beziehungen zu Unternehmen aufgebaut zu haben, hilft es, eine Liste mit potenziellen Firmenpartnern zu erstellen. Recherchieren Sie genau, welches Unternehmen in welchem Bereich tätig ist, welche Marketing-Strategien dort verfolgt werden, wer der richtige Ansprechpartner für das eigene Anliegen ist etc. (vgl. Kasten »Wie man mit System recherchiert « Seite 228).

Bei der gezielten Auswahl potenzieller Sponsoren dienen Größe und Standort des Unternehmens als erste Anhaltspunkte. Passt zu unserem Projekt oder Verein eher ein großer Konzern oder ein inhabergeführtes mittelständisches Unternehmen? Wenn Sie beispielsweise Unterstützer für ein Projekt mit arbeitslosen Jugendlichen suchen, lohnt es eher, mittelständische Unternehmen vor Ort anzusprechen. Ein weltweit agierendes Unternehmen wird auch eher eine international ausgerichtete soziale Organisation bevorzugen, wenn es beispielsweise darum geht, eine gemeinsame Kampagne gegen Sextourismus in einschlägigen Ländern umzusetzen. 
Und natürlich stellt sich die Frage auch umgekehrt. Jede Organisation muss für sich definieren, welches Unternehmen zum eigenen Image passt und wie eine Kooperation sinnvoll gestaltet werden kann. Bei der Suche nach dem richtigen Unternehmen stellen sich demnach folgende Fragen:

- Welches Unternehmen hat aufgrund seines Profils oder Images eine Affinität zu unserer Organisation oder unserem Projekt?

- Welche Firmen haben ihren Sitz am Ort oder in der Region?

- Welche Branche passt zum Projekt oder zur Organisation?

- Welche Firmen passen von der Größe zur Organisation und zum Etat?

- Welche Produkte und Marken sind kompatibel?

- Welche Firmen sprechen welche Zielgruppen an?

\section{Der Kontaktaufbau zu Unternehmen}

Neben einem überzeugenden Projekt oder "Sponsoring-Produkt « sind die guten Kontakte zu den zuständigen Verantwortlichen entscheidend. Je persönlicher und besser die Beziehungen, desto erfolgreicher die Zusammenarbeit.

Eigenes Kontaktpotenzial ermitteln: Zunächst sollte also systematisch analysiert werden, über welche Kontakte die Organisation bereits verfügt. Gibt es in den eigenen Reihen schon persönliche Verbindungen zu Entscheidungsträgern oder zu Menschen, die solche Kontakte vermitteln können? Häufig ist sich eine Organisation ihres eigenen Potenzials nicht bewusst. Dann ist es sinnvoll, in einem Workshop oder gemeinsamen Brainstorming diese Kontakte zu ermitteln und transparent zu machen. Die Ergebnisse sind oft erstaunlich und gleichen einer wahren Fundgrube. Darüber hinaus empfiehlt es sich, über eigene Aktivitäten, beispielsweise Pressearbeit oder Veranstaltungen, Interesse zu wecken und eigene Netzwerke aufzubauen. Gute Unternehmenskontakte müssen mühevoll aufgebaut werden. Aber das ist eine Investition, die sich langfristig lohnt, auch wenn sie sich nicht immer gleich beim ersten Projekt auszahlt.

Unternehmensansprache planen: Sind persönliche Kontakte nicht vorhanden, bleibt nur der steinige Weg der Akquisition: die Neuanbahnung von Unternehmenskontakten. Besonders bei der »Kaltakquisition « (Neuansprache) sollten Sie sich gut vorbereiten. Vor der ersten Kontaktaufnahme im Dienste Ihres Sponsoring-Projektes muss der zuständige Ansprechpartner im Unternehmen herausgefunden werden. Bei kleineren Unternehmen ist das meistens der Inhaber selbst oder der Geschäftsführer. Große Unternehmen beschäftigen Marketingund Öffentlichkeitsabteilungen. In welcher Abteilung das Sponsoring angesiedelt ist, müssen Sie oft erst herausfinden. Und den Namen des zuständigen Entscheiders sollten Sie unbedingt kennen.

Telefonisch Kontakt aufbauen: Ziel der ersten Kontaktaufnahme ist es, Interesse für das Projekt zu wecken und die Erlaubnis zu bekommen, das Exposé schikken zu können. Bevor Sie sich zum Sponsoring-Verantwortlichen durchstellen lassen, ist es daher sinnvoll, einen Telefon-Leitfaden zu erstellen. Das gibt Sicherheit, wenn man aufgeregt ist und hilft, sich auf das Wesentliche zu konzentrieren. Natürlich ist es nicht mit einem einzigen Kontakt getan. Wenn Sie das Exposé verschickt haben, fragen Sie nach, ob es auch angekommen ist. Freundliches, aber dennoch beharrliches Nachfassen führt oftmals zum Ziel: Besteht weiterhin Interesse? Noch weitere Informationen gewünscht? Wenn irgend möglich, vereinbaren Sie einen Termin zur persönlichen Präsentation des Projekts.

Angebot schriftlich skizzieren: Um dem Unternehmen Ihr Angebot konkret vorzustellen, ist eine überzeugende Projektdarstellung oder sogar die Kreation eines »Sponsoring-Produktes « erforderlich. Das Konzept wird in einem Exposé zusammengefasst, das interessierten Unternehmen übersandt wird (vgl. Kasten »Wie man ein Exposé schreibt«).

Richtigen Zeitpunkt wählen: Bei den meisten Firmen ist das Geschäftsjahr identisch mit dem Kalenderjahr und die Budgetplanungen für das nächste Jahr erfolgen in der Regel im Herbst. Zu diesem Zeitpunkt werden auch die Sponsoringbudgets und PR-Etats festgelegt. Auch wenn Ausnahmen möglich sind, sollte sich eine Organisation auf diese Abläufe einstellen und spätestens nach der Sommerpause mit Sponsoring-Projekten auf die Unternehmen zugehen. Entsprechend langfristig sollten Sie die eigenen Projekte planen und eine Vorlaufzeit von sechs bis acht Monaten einkalkulieren.

Die Akquisition von Unternehmenspartnern ist nicht einfach. In der Regel müssen Sie eine Vielzahl von Firmen kontaktieren, bis Sie auf konkretes Interesse stoßen. Und auf dem Weg zum Ziel wer-

\section{Wie man ein Exposé schreibt}

Das Exposé enthält mehrere Komponenten:

- die Selbstdarstellung der Organisation unter Hervorhebung aller Stärken und Möglichkeiten, wie Anzahl der Fördermitglieder, Kontakte zu Medien oder prominente Unterstützer etc.

- die Beschreibung des geplanten Projekts mit Zielsetzung und Maßnahmen, angesprochenen Zielgruppen und den Besonderheiten (Anknüpfungspunkte und zu erwartender »Return on Investment « für Unternehmen)

- das Angebot an den Sponsor, die kommunikativen Gegenleistungen, die erbracht werden können - Werbemöglichkeiten, PR-Maßnahmen, Veranstaltungen etc. Hier ist vor allem der kommunikative Nutzen für den Sponsor relevant und entsprechend herauszustellen.

- den »Preis «, also die erwünschten Leistungen eines oder mehrerer Unternehmen in Form von Geld, Sachleistungen, Personaleinsatz etc.

Nicht zuletzt sollte das Exposé »kurz und knackig « formuliert und ansprechend gestaltet sein. Manager haben in der Regel wenig Zeit. Sie wollen auf den ersten Blick erkennen, worum es geht und worin für sie der Nutzen liegt.

Helene Reuther

morgenwelt, agentur für social marketing gmbh

Brüsseler Straße 21, 50674 Köln, Telefon 0221 931-8890, Fax 0221 931-88999

E-Mail H.Reuther@morgenwelt.net

Internet http://www.morgenwelt.net 
den Sie überwiegend Absagen kassieren. Die Faustregel lautet: Auf eine Zusage kommen 40 Absagen und mehr! Lassen Sie sich trotzdem nicht entmutigen, aber bleiben Sie realistisch in Ihrer Einschätzung.
Definieren Sie die eigenen Ziele und Grenzen: Überlegen Sie im Vorfeld: Was wollen Sie mit der Unternehmenspartnerschaft erreichen? Was ist Ihnen unbedingt wichtig? Wo liegt Ihre Schmerzgrenze? Worauf wollen Sie sich auf keinen Fall

\section{»Der Aufbau guter Unternehmens- kontakte ist mübevoll - aber die Investition lobnt sich «}

Die Mühe hat sich gelohnt, Sie haben es geschafft. Das Unternehmen will als Sponsor oder Kooperationspartner einsteigen. Jetzt geht es um »die Wurst «. Sie müssen die Verhandlung optimal vorbereiten, denn wie so oft gilt auch hier: 80 Prozent des Erfolgs liegt in der sorgfältigen Vorbereitung der Verhandlung.

Erforschen Sie gründlich die Interessen der anderen Seite: Bevor Sie in die Verhandlung gehen, sollten Sie sich intensiv mit den Interessen des potenziellen Partners beschäftigen. Sammeln Sie so viele Informationen wie möglich über die Marketing- und Kommunikations-Ziele, aktuellen PR-Aktivitäten, Image und Produkte des Unternehmens.

\section{Wie man mit System} recherchiert

Wenn man gezielt nach bestimmten Unternehmen oder Daten sucht, ist das Internet eine gute und schnelle Informationsmöglichkeit. Für die weitere Recherche generell in Frage kommender Unternehmen sind Branchen- und Markenhandbücher, Verzeichnisse von Industrie- und Handelskammern, Messekataloge, Wirtschaftszeitschriften etc. hilfreiche Informationsquellen. Hinweise auf das bisherige Förderverhalten eines Unternehmens müssen durch regelmäßige Zeitungslektüre (vor allem des Wirtschaftsteils!), die aufmerksame Beobachtung von Werbung (Anzeigen, Plakate, Flyer etc.) und mit Hilfe persönlicher Kontakte zusammengetragen werden.

Helene Reuther einlassen? Wie lautet »Plan B«? Also: Was ist Ihre Alternative, wenn Sie sich nicht einigen können?

Legen Sie Ihre Gesprächsstrategie fest: Spielen Sie in Gedanken das Gespräch durch. Womit beginnen Sie? Wie verhalten Sie sich bei kritischen Fragen? Es empfiehlt sich, die Verhandlung zu zweit zu führen. Dann kann man sich gegenseitig entlasten oder die Bälle zuwerfen. Klären Sie aber vorher die Rollenverteilung. Legen Sie fest, wer welche Aufgabe übernimmt und für welchen Part zuständig ist.

Wählen Sie die besten Verhandlungspartner aus: In jeder Gruppe oder Organisation gibt es Menschen mit unterschiedlichen Fähigkeiten. Wer sind bei Ihnen die geeigneten Verhandlungsführer? Wer ist verhandlungssicher und kann Ihre Interessen am besten vertreten? Wer ist rhetorisch besonders geschickt? Wer kann persönlich und verbindlich auftreten, lässt sich aber nicht leicht einschüchtern?

Ziele und Interessen in Übereinstimmung bringen: Nun geht es konkret darum, die unterschiedlichen Positionen und Interessen - die der eigenen Organisation und die des potenziellen Unternehmenspartners - im Gespräch auszuloten und in Einklang zu bringen.

Treten Sie klar und verbindlich auf. Auch wenn man manchmal das Gefühl hat, in der schwächeren Position zu sein: Verhalten Sie sich nicht wie ein Bittsteller, sondern als selbstbewusster Partner. Gleichzeitig sollten Sie auch Verständnis für die andere Seite zeigen sowie deren Nöte und Zwänge. Ziel jeder Verhandlung ist es, eine »Win-win-Lösung « zu erzielen. Beide Parteien sind gemeinsam dafür verantwortlich, das bestmögliche und für beide Seiten tragfähige Ergebnis zu erzielen.

\section{Fazit}

Bei der Suche nach Unternehmenspartnern muss ein Sponsoringprojekt in mindestens einem der drei folgenden Bereiche punkten:

- Das Projekt ist so überzeugend, dass ein Verantwortlicher im Unternehmen die damit verbundenen Chancen sofort erkennt und von ihm begeistert ist.

- Das Projekt hat ein stichhaltiges Kommunikationskonzept.

- Die Organisation verfügt über gute Kontakte zu den Entscheidern an der Unternehmensspitze oder die Organisation ist selbst so bedeutend, dass sich ihr leicht die Türen zu den oberen Etagen eines Unternehmens öffnen.

Ist das Projekt weder besonders überzeugend, noch die begleitende Kommunikation ein Highlight, können allenfalls noch intensive Kontakte zur Vorstandsebene helfen. Wird keines der drei Kriterien richtig erfüllt, sollte man sich sehr überlegen, ob sich die erheblichen Aufwendungen - personell und finanziell - für die Sponsorensuche wirklich lohnen.

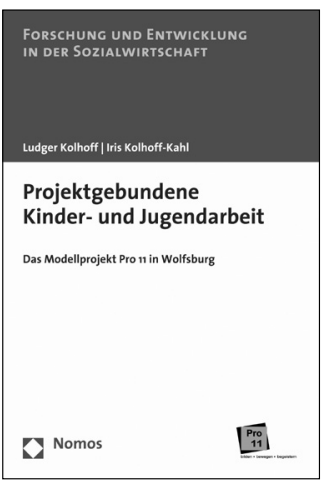

\section{Projektgebundene} Kinder- und Jugendarbeit

Das Modellprojekt Pro 11 in Wolfsburg

Von Prof. Dr. Ludger Kolhoff und Prof. Dr. Iris Kolhoff-Kahl 2008, 135 S., brosch., 24,-€, ISBN 978-3-8329-3733-1 (Forschung und Entwicklung in der Sozialwirtschaft, $B d .5$ )

Nomos 
"Wenn Sie einen Dollar in Ihr Unternehmen stecken wollen, so müssen Sie einen zweiten bereithalten, um das bekanntzugeben." Henry Ford, amerikanischer Industrieller (1863-1947)

„Ein wichtiges Merkmal eines realistischen und systemorientierten Unternehmungsbildes ist die Tatsache, dass eine Unternehmung nicht jene strenge Rationalität aufweist, die ihr in der klassischen Betriebswirtschaft unterstellt wird."

Prof. Dr. Fredmund Malik, österreichischer Managementberater

„Was man nicht kommunizieren kann, kann man auch nicht realisieren."

Dr. Friedmar Nusch, Leiter Unternehmenskommunikation Heidelberger Druckmaschinen AG

"Ein Unternehmen ist kein Zustand, sondern ein Prozess. " Ludwig Bölkow, deutscher Ingenieur und Unternehmer (1912-2003)

„Organisationen halten Leute beschäftigt, unterhalten sie bisweilen, vermitteln ihnen eine Vielfalt von Erfahrungen, halten sie von den Straßen fern, liefern Vorwände für Geschichtenerzählen und ermöglichen Sozialisation. Sonst haben sie nichts anzubieten."

Karl E. Weick, amerikanischer Soziologe (geb. 1936)

"Nur weil Sie schon zweimal Konkurs gemacht haben, können Sie doch nicht behaupten, Sie hätten Erfahrung im Management von Non-Profit-Organisationent"

Oswald Huber, österreichischer Psychologe und Karikaturist (geb. 1942)

"Mit keinem noch so schönen oder logischen Geschäftsplan wird ein Unternehmen die Loyalität der Menschen gewinnen, denen dieser Plan aufgezwungen wird, und zwar einfach deshalb, weil die Beschäftigten an seiner Aufstellung nicht beteiligt waren."

Richard Sennett, amerikanischer Soziologe (geb. 1943) 